\title{
Validation of an LC-MS/MS Method for the Determination of Propofol, Midazolam, and Carbamazepine in Rat Plasma: Application to Monitor Their Concentrations Following Co-administration
}

\author{
Mohamed Abo El Hamd, ${ }^{a}$ Mitsuhiro Wada, ${ }^{*, b}$ Rie Ikeda, ${ }^{a}$ Shigeru Kawakami, ${ }^{a}$ and \\ Kenichiro Nakashima ${ }^{c}$ \\ ${ }^{a}$ Graduate School of Biomedical Sciences, Nagasaki University; 1-14 Bunkyo-machi, Nagasaki 852-8131, Japan: \\ ${ }^{b}$ Faculty of Pharmaceutical Sciences, Kyushu University of Health and Welfare; 1714-1 Yoshino-cho, Nobeoka, \\ Miyazaki 882-8508, Japan: and ${ }^{c}$ Faculty of Pharmaceutical Sciences, Nagasaki International University; \\ 2825-7 Huis Ten Bosch, Sasebo, Nagasaki 859-3298, Japan. \\ Received March 2, 2015; accepted May 12, 2015
}

Propofol (PRO) is a hypnotic used to induce and maintain general anesthesia. A risk of drug-drug interactions exists in cases of clinical co-administration of PRO and midazolam (MDZ) or carbamazepine (CBZ). Therefore a sensitive and rapid assay is needed to monitor these drugs. In this study, a sensitive and selective liquid chromatography-tandem mass spectrometry technique was developed for simultaneous determination of PRO, MDZ, and CBZ in plasma. Simultaneous selected reaction monitoring in the positive and negative ionization modes was used for mass detection. Analytes were isolated from plasma samples by a simple, economic, and rapid solid-phase extraction method. Chromatographic separations were achieved using a Chromolith Performance RP-18e analytical column $(100 \times 4.6 \mathrm{~mm}$ i.d.) with a mixture of acetonitrileammonium acetate buffer $(10 \mathrm{~mm}, \mathrm{pH} 3.5)(90: 10, \mathrm{v} / \mathrm{v})$ as the mobile phase. The method was fully validated for PRO, MDZ, and CBZ over concentrations ranging at $1-100,2-100$, and $7-1000 \mathrm{ng} / \mathrm{mL}$, respectively, with acceptable validation parameters. Furthermore, the method was applied to monitor PRO and MDZ or CBZ following co-administration in rats. plasma

Key words propofol (PRO); midazolam (MDZ); carbamazepine (CBZ); pharmacokinetic interaction; rat

Propofol (PRO) is an intravenous (i.v.) hypnotic agent used to induce and maintain general anesthesia.) Owing to its desirable antiemetic, anticonvulsant, and antioxidant properties, PRO is commonly used in total i.v. anesthesia. ${ }^{2)}$ PRO does not show cumulative effects; hence, its anesthetic effect can be prolonged by repeated injections or continuous infusion. ${ }^{3)}$ Carbamazepine (CBZ) is a dibenzazepine tricyclic drug used for the treatment of epilepsy, trigeminal neuralgia, and schizophrenia. ${ }^{4-6)}$ Epilepsy is of concern in anesthesia due to the possibility of interactions between antiepileptic drugs (AEDs) and co-administered agents, particularly with respect to postoperative seizures and the intensive care management of status epilepticus. ${ }^{7}$ Benzodiazepines such as midazolam (MDZ), which has hypnotic, muscle-relaxant, anticonvulsant, and anxiolytic properties, is commonly used to induce anesthesia and to treat generalized seizures or status epilepticus. ${ }^{8}$ Individually, both PRO and CBZ are known to either inhibit or induce a variety of hepatic cytochrome enzymes. ${ }^{9,10)}$ The important role of the anesthesiologist is to minimize the risk of drug-drug interactions while providing anesthesia that is adequate to the surgical conditions. Moreover, a thorough understanding of potential pharmacokinetic (PK) interactions between drugs that are used for anesthesia is essential. In our pervious study, we reported an interaction between two drugs that are typically co-administered during general anesthesia: PRO and the narcotic analgesic remifentanil. ${ }^{11)}$

In this study, a liquid chromatography-tandem mass spectrometry (LC-MS/MS) method for the simultaneous determination of $\mathrm{PRO}, \mathrm{MDZ}$, and $\mathrm{CBZ}$ in rat plasma was developed, validated, and applied to monitor these drugs following co- administration in rat.

\section{MATERIALS AND METHODS}

Chemicals and Reagents PRO, MDZ, ammonium acetate, acetic acid, and acetonitrile were obtained from Wako Pure Chemical Industries, Ltd. (Osaka, Japan). CBZ was obtained from Sigma-Aldrich, Inc. (St. Louis, MO, U.S.A.), and all other reagents were of analytical reagent grade. Water was deionized and distilled using an Aquarius GSR-500 automatic water distillation apparatus (Advantec, Tokyo, Japan). PRO, MDZ, and CBZ stock solutions at a concentration of $1 \mathrm{mg} / \mathrm{mL}$ were prepared by dissolving an appropriate amount of drug in acetonitrile. Stock solutions were stored at $4^{\circ} \mathrm{C}$ until used.

LC-MS/MS System and Conditions A Waters 2695 separation module (Waters Co., Milford, MA, U.S.A.) was used for analyte separation. A variable negative- and positiveionization Quattro micro ${ }^{\mathrm{TM}}$ triple-quadrupole mass spectrometer (Waters) equipped with an electrospray ionization (ESI) source was used for mass analysis. Chromatographic separations were achieved using a Chromolith Performance RP-18e analytical column $(100 \times 4.6 \mathrm{~mm}$ i.d.; Merck, Darmstadt, Germany) kept at $30^{\circ} \mathrm{C}$. The mobile phase consisted of a mixture of acetonitrile-ammonium acetate buffer (10 mm, $\mathrm{pH} 3.5)$ $(90: 10 \%, v / v)$ pumped at a flow rate of $0.5 \mathrm{~mL} / \mathrm{min}$. Selected reaction monitoring (SRM) mode was used for MS analyses, which were performed simultaneously in negative-ion mode for PRO and positive-ion mode for MDZ and CBZ. Other MS/MS parameters are shown in Supplementary Table 1.

Pretreatment of Plasma Samples A $20-\mu \mathrm{L}$ volume of 
MDZ or CBZ solution (as an internal standard; $50 \mathrm{ng} / \mathrm{mL}$ ) was added to a $200-\mu \mathrm{L}$ aliquot of rat plasma and the sample was then vortexed briefly. A Bond Elut-C18 cartridge (100 mg, $1 \mathrm{~mL}$, Agilent Technologies Inc., Wilmington, DE, U.S.A.) was conditioned with methanol $(1 \mathrm{~mL})$ and deionized water $(1 \mathrm{~mL})$ and then the sample was loaded onto the cartridge. The cartridge was washed twice with $500 \mu \mathrm{L}$ of $10 \%$ methanol and the analytes were eluted with $400 \mu \mathrm{L}$ of methanol. Next, $20 \mu \mathrm{L}$ of the eluate was injected directly into the LC-MS/MS for analysis. Triplicate measurements for each sample were performed, and the amount of analyte recovered is reported as the mean and standard deviation (S.D.).

Method Validation The method was validated according to the U.S. Food and Drug Administration Guideline for Bioanalytical Methods as detailed below. ${ }^{12)}$ The selectivity of the proposed method for any interference was estimated by analyzing 10 different blank rat plasma samples. Two calibration curves for PRO were prepared (using samples spiked with $20 \mu \mathrm{L}$ of $50 \mathrm{ng} / \mathrm{mL} \mathrm{CBZ} \mathrm{or} \mathrm{MDZ} \mathrm{as} \mathrm{ISs),} \mathrm{and}$ five concentrations were used for the PRO, MDZ, and CBZ calibration curves. The limit of detection (LOD) and limit of quantification (LOQ) were defined as the concentration of analyte yielding a peak with a signal to noise ratio of 3 and 10, respectively. The method's accuracy and precision were assessed by analyzing quality control (QC) plasma samples spiked with three concentrations on five different validation days. Accuracy is expressed as a percentage of the calculated concentration divided by the nominal concentration. Both intra- and inter-day precision were evaluated and were determined as the relative standard deviation (R.S.D.). Matrix effects (MEs) were quantified as the internal standard (IS)normalized matrix factor (IS-MF), which is the ratio of the peak area in the presence of matrix for each plasma sample divided by the mean peak area ratio in the absence of matrix. Analyte recovery was determined by comparing the peak area for each extracted analyte spiked at three concentrations to that of the corresponding non-extracted pure standard (at the same concentrations $[n=5])$.

Stability of Analytes in Plasma The stability of the analytes was assessed by analyzing low-, mid-, and highconcentration QC samples (with five measurements for each concentration) exposed to different storage and temperature conditions. The stability of analytes was assessed after three freeze-thaw cycles, storage at room temperature $\left(25^{\circ} \mathrm{C}\right)$ for $4 \mathrm{~h}$, and storage of extracted samples for $12 \mathrm{~h}$ at room temperature.

Application to Monitor the Drugs Following Coadministration The plasma samples used in the present study were obtained from male Wistar rats (weight $=250-320 \mathrm{~g}$ ) purchased from Kyudo Experimental Animal (Saga, Japan). All animal procedures and care protocols were approved by the Nagasaki University Animal Care and Use Committee (No. 1406021153). Rats were anesthetized with ethyl carbamate (1.5 g/kg; intraperitoneal (i.p.) injection) and two indwelling cannulae (a femoral vein cannula for drug administration and a femoral artery cannula for blood sampling) were placed in each animal. For the co-administration study, PRO $(5 \mathrm{mg} / \mathrm{mL})$, MDZ $(2 \mathrm{mg} / \mathrm{mL})$, and CBZ $(5 \mathrm{mg} / \mathrm{mL})$ solutions were prepared in Intralipid ${ }^{\circledR} 10 \%$ emulsion (Fresenius Kabi, Tokyo, Japan). PRO was administered by i.v. infusion for $60 \mathrm{~min}$ using a microinjection pump (CMA/100; Carnegie
Medicine, Stockholm, Sweden); MDZ and CBZ were administered i.p. with an injection volume of $1 \mathrm{~mL} / \mathrm{kg}$ of body weight. Following administration of PRO, MDZ, and CBZ, blood samples $(0.35 \mathrm{~mL}$ each) were drawn at various intervals between 5 and $240 \mathrm{~min}$. Plasma samples were prepared by centrifugation $(2000 \times \boldsymbol{g})$ of whole blood for $10 \mathrm{~min}$ at $4^{\circ} \mathrm{C}$. Plasma samples were stored at $-30^{\circ} \mathrm{C}$ until analysis.

\section{RESULTS}

Optimization of Assay Conditions The method allowed for ready switching between negative- and positive-ionization LC-MS/MS modes; PRO was determined in negative ESI mode and MDZ and CBZ were determined in positive ESI mode. In full-scan mass spectra, predominant molecular ions $\left([\mathrm{M}-\mathrm{H}]^{-}\right.$or $\left.[\mathrm{M}+\mathrm{H}]^{+}\right)$were observed at $m / z 176.94,237.07$, and 326.74, representing $\mathrm{PRO}, \mathrm{CBZ}$, and MDZ, respectively. The most abundant product ions observed were at $\mathrm{m} / \mathrm{z}$ 176.94, 194.02, and 291.9 for PRO, CBZ, and MDZ, respectively (Supplementary Figure 1). In the present study, the false $\mathrm{m} / \mathrm{z}$ transition $176.94 \rightarrow 176.94$ for PRO determination in SRM was used because of the low ratio of the intensities of the product to parent ions at the true transition $(\mathrm{m} / \mathrm{z} 176.94 \rightarrow 160.87)$. Complete separation of all analytes was achieved within $5 \mathrm{~min}$.

Method Validation Calibration curves for PRO, MDZ, and $\mathrm{CBZ}$ in rat plasma were prepared over the concentration ranges $1-100,2-100$, and $7-1000 \mathrm{ng} / \mathrm{mL}$ for $\mathrm{PRO}, \mathrm{MDZ}$, and CBZ, respectively. The IS peak area ratios of all three analytes exhibited good linearity over the selected concentration ranges, with correlation coefficients $(r) \geq 0.991$. No endogenous matrix peaks were observed at the retention times of the analytes in any of the evaluated plasma samples (Supplementary Figures 2 and 3). The sensitivity of the method for PRO, MDZ, and CBZ was sufficient for their determination in rat plasma samples at doses relevant to those used in clinical application in humans. The intra-day precision for each analyte ranged between 1.7 and $14.3 \%$, whereas the inter-day precision ranged between 5.1 and $18.3 \%$, and the accuracy ranged between 98.6 \pm 4.4 and 109.8 $\pm 11.0 \%$ (Supplementary Table 2). The mean recoveries of PRO, MDZ, and CBZ were 94.9 \pm 5.9 , $88.5 \pm 7.9$, and $84.6 \pm 7.7 \%$ and were similar at all analyte concentrations, without significant concentration dependence. The IS-MF as calculated for the present work showed no significant ion suppression or enhancement at low, mid, or high concentrations of PRO, MDZ, and CBZ. The RSD\% ranged from 1.9 to $11.9 \%$ for all three analytes (Supplementary Table 3).

The stability of PRO, MDZ, and CBZ in rat plasma stored under different conditions was examined (Supplementary Table 4). No significant degradation occurred under the conditions used in this study, as the concentration deviated by no more than $19.7 \%$ relative to the reference nominal concentration for all three analytes. All three analytes could be safely stored at room temperature for $4 \mathrm{~h}$ or stored at $-30^{\circ} \mathrm{C}$ and subjected to three freeze-thaw cycles. In addition, analysis of the QC samples following solid-phase extraction (SPE) showed no significant degradation of the analytes after storage of the extracts for $12 \mathrm{~h}$ at room temperature.

Application to Monitor the Drugs Following Co-administration The applicability of the proposed method was evaluated by monitoring drugs after co-administration of PRO and MDZ. Plasma concentration-time profiles for PRO, MDZ, 
(A)

(B)

(C)
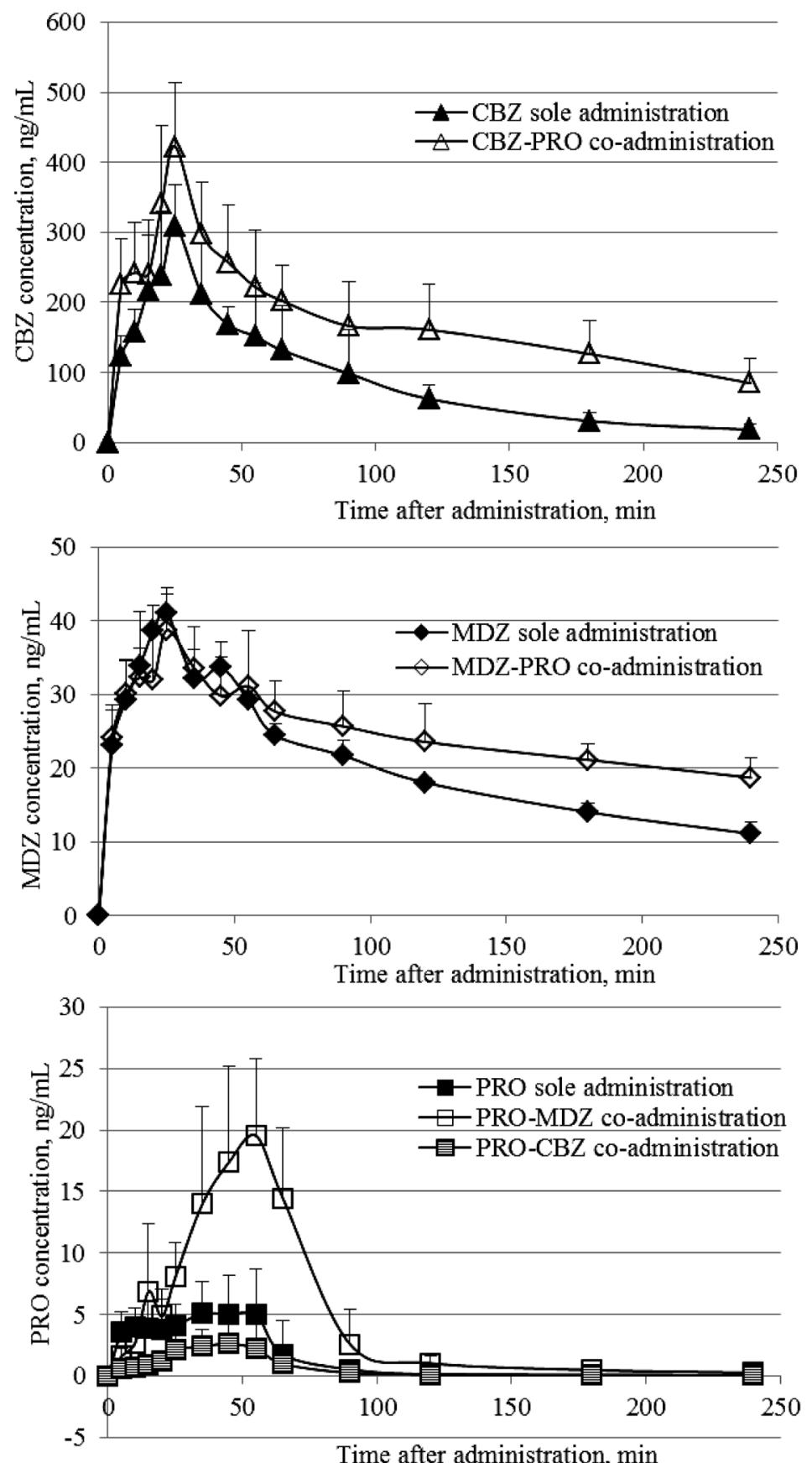

Fig. 1. Plasma Concentration-Time Profiles for PRO (A), MDZ (B), and CBZ (C)

Data are expressed as the mean + S.D. $(n=3)$.

and $\mathrm{CBZ}$ after sole and co-administration are shown in Figs. 1 (A)-(C). Sole or co-administration of PRO with MDZ and/or CBZ was evaluated through $60 \mathrm{~min}$ of i.v. infusion and then until $240 \mathrm{~min}$ after the infusion device was stopped.

After the first $10 \mathrm{~min}$ of the infusion process, the concentration of PRO was stable. The mean area under the curve $\left(A U C_{10-240}\right)$ for sole administration $(n=6)$ was $215.8 \pm 37.7 \mathrm{ng} /$ $\mathrm{mL} \times \mathrm{min}$. The $A U C \mathrm{~s}_{10-240}$ for PRO after co-administration with MDZ and CBZ was 455.2 \pm 118.5 and $79.5 \pm 39.9 \mathrm{ng} /$ $\mathrm{mL} \times \min$, respectively.

The plasma concentration of PRO increased significantly after co-administration with MDZ $(p=0.015)$ and decreased significantly after co-administration with CBZ $(p=0.013)$. For $\mathrm{MDZ}$, the $A U C_{0-240}\left(\times 10^{3} \mathrm{ng} / \mathrm{mL} \times \mathrm{min}\right)$ was $4.9 \pm 0.2$ after sole administration versus $6.3 \pm 0.2$ after co-administration, and for $\mathrm{CBZ}$, the $A U C_{0-240}\left(\times 10^{3} \mathrm{ng} / \mathrm{mL} \times \mathrm{min}\right)$ was $21.5 \pm 7.0$ after sole administration versus $41.7 \pm 11.9$ after co-administration.

The plasma concentrations of both PRO and MDZ increased after co-administration, which could be related to PRO-mediated inhibition of hepatic CYP3A isoforms, resulting in decreased clearance of MDZ. ${ }^{13,14)}$ Another possible explanation is that hemodynamic changes led to an increase in the PRO plasma concentration. No other reports of studies investigating the interaction between $\mathrm{PRO}$ and $\mathrm{CBZ}$ during anesthesia in humans or animals have been published. The basis for studying the interaction between PRO and CBZ here was the common metabolic pathway involving the isoenzymes CYP3A4 and CYP2C8. CBZ is an inducer of several 
potential pathways of drug elimination, including pathways mediated by CYP1A2, CYP2C9, and CYC3A4, as well as the active transporter P-glycoprotein. ${ }^{15)}$ Therefore, the metabolism of drugs that are metabolized by these CYPs, such as PRO, is likely to be affected by CBZ co-administration. ${ }^{15,16)}$ The results of this study revealed that the co-administration of PRO and CBZ leads to a significant $(p=0.013)$ decrease in the plasma concentration of PRO. This finding is consistent with reports in the literature showing that unlike other AEDs, CBZ is an inducer of several potential drug elimination pathways.

The importance of these findings should not be underestimated, as PRO is one of the most common hypnotics used in general anesthesia. Moreover, CBZ is the most commonly used AED and is also indicated for treating trigeminal neuralgia and migraine headache; thus, a significant percentage of people require this drug at some point in their life. ${ }^{17)}$ However, further confirmation of these findings in studies using a suitable number of human samples is needed before the method is applied in the patients-care setting.

In conclusion, we developed and validated the LC-MS/MS method for the rapid and simultaneous determination of PRO, MDZ, and CBZ in rat plasma. We found that the plasma pretreatment procedure described here is simple, rapid, and selective for extracting these analytes from plasma. In addition, the applicability of the proposed LC-MS/MS method could be shown to monitor the concentrations of PRO and MDZ or CBZ following co-administration.

Conflict of Interest The authors declare no conflict of interest.

Supplementary Materials The online version of this article contains supplementary materials.

\section{REFERENCES}

1) Miekisch W, Fuchs P, Kamysek S, Neumann C, Schubert JK. Assessment of propofol concentrations in human breath and blood by means of HS-SPME-GC-MS. Clin. Chim. Acta, 395, 32-37 (2008).

2) Ghoneim MM, Korttila K. Pharmacokinetics of intravenous anaesthetics: duplications for cinical use. Clin. Pharmacokinet., 2, 344-372 (1977).
3) Ragno G, Cicinelli E, Schonauer S, Vetuschi C. Propofol assay in biological fluids in pregnant women. J. Pharm. Biomed. Anal., 15, 1633-1640 (1997).

4) Bernus I, Dickinson R, Hooper W, Eadie M. Dose-dependent metabolism of carbamazepine in humans. Epilepsy Res., 24, 163-172 (1996)

5) Deleu D, Aarons L, Ahmed IA. Population pharmacokinetics of free carbamazepine in adult Omani epileptic patients. Eur. J. Clin. Pharmacol., 57, 243-248 (2001).

6) Albani F, Riva R, Baruzzi A. Carbamazepine clinical pharmacology: A review. Pharmacopsychiatry, 28, 235-244 (1995).

7) Gratrix A, Enright S. Epilepsy in anaesthesia and intensive care. Contin. Educ. Anaesth. Crit. Care Pain, 5, 118-121 (2005).

8) Nordt SP, Clark RF. Midazolam: a review of therapeutic uses and toxicity. J. Emerg. Med., 15, 357-365 (1997).

9) Guitton J, Buronfosse T, Desage M, Flinois JP, Perdrix JP, Brazier JL, Beaune P. Possible involvement of multiple human cytochrome P450 isoforms in the liver metabolism of propofol. Br. J. Anaesth., 80, 788-795 (1998).

10) Johannessen Landmark C, Patsalos PN. Drug interactions involving the new second- and third-generation antiepileptic drugs. Expert Rev. Neurother., 10, 119-140 (2010).

11) El Hamd MA, Wada M, Ikeda R, Kawakami S, Kuroda N, Nakashima K. Simultaneous determination of propofol and remifentanil in rat plasma by liquid chromatography-tandem mass spectrometry: application to preclinical pharmacokinetic drug-drug interaction analysis. Biomed. Chromatogr., 29, 325-327 (2015).

12) Food and Drug Administration (FDA). Guidance for industry. Bioanalytical method validation U.S. Department of Health and Human Services, pp. 1-25 (2013).

13) Hamaoka N, Od Y, Has I, Mizutan K, Nakamot T, Ishizak T, Asad A. Propofol decreases the clearance of midazolam by inhibiting CYP3A4: an in vivo and in vitro study. Clin. Pharmacol. Ther., 66, 110-117 (1999).

14) Vuyk J, Lichtenbelt BJ, Olofsen E, van Kleef JW, Dahan A. Mixedeffects modeling of the influence of midazolam on propofol pharmacokinetics. Anesth. Analg., 108, 1522-1530 (2009).

15) John R, Phillip H. "Carbamazepine: Watch for many potential drug interactions.”: 〈http://www.hanstenandhorn.com/hh-article02-08.pdf), cited 25 Oct., 2014.

16) Anderson J, Moor CC. Anti-epileptic drugs: a guide for the nonneurologist. Clin. Med., 10, 54-58 (2010).

17) Medhi B, Singh PK, Prakash A, Avti P. Effect of esomeprazole on the pharmacokinetics of carbamazepine. Indian J. Pharmacol., 43, 73-75 (2011). 